Rev. Latinoam. Psicopat. Fund., São Paulo, v. 14, n. 1, p. 166-189, março 2011

\title{
Delirium e confusão mental no século XIX: uma história conceitual ${ }^{*}$
}

\author{
German E. Berrios
}

O delirium permaneceu uma categoria psiquiátrica estável até o início do século XIX, quando passou por uma redefinição etiológica e fenomenológica, precipitando a transformação das insanidades funcionais em psicoses.

A confusão, introduzida pelos franceses ao longo da segunda metade do século, referia-se a uma síndrome mais ampla (porém incluindo) o delirium. Enfatizava o pensamento caótico e as falhas cognitivas. A noção de turvação da consciência (e desorientação temporoespacial) estabeleceu um denominador comum para as duas concepções, enquanto Chaslin e Bonhoeffer redefiniram a confusão e o delirium como as manifestações estereotipadas da insuficiência cerebral aguda.

Palavras-chave: Delirium - história; confusão mental - história;

transtornos da consciência - história; transtornos cognitivos - história

* Publicado originalmente em Brit. J. Psychiat., n. 139, p. 439-449, 1981. Tradução de Lazslo A. Ávila e revisão técnica de Ana Maria G. R. Oda. 


\section{Parte 1: Delirium}

Delirium é um conjunto de sintomas mentais e comportamentais que ocorrem na esteira de doenças físicas (Lipowski, 1980). As definições em geral ressaltam sua natureza transitória, intermitente e estereotipada. Essa última característica (que provavelmente reflete a base biológica do delirium) subjaz a sua secular estabilidade fenomenológica e fornece aos historiadores um point de repère clínico.

Este artigo vai explorar as relações entre o delirium, a confusão e a teoria psicológica, e seu papel na formação de uma taxonomia psiquiátrica viável; e argumentará que o delirium e não a paralisia dos alienados (Bayle, 1825; Zilboorg, 1941; Leibbrand e Wettley, 1961; Ackerknecht, 1957), forneceu o paradigma descritivo e organizacional para a psiquiatria do século XIX. Do ponto de vista historiográfico, assumiremos que a psiquiatria atual compartilha do mesmo espaço epistemológico que sua contraparte do século XIX.

\section{Os primórdios}

Referências a uma associação entre as doenças físicas e mentais podem ser encontradas nos escritos médicos desde a antiguidade. Entre os gregos, por exemplo, os termos assemelhados delirium, frenite e letargia, referiam-se a graves perturbações do pensamento, humor e ação associados à doença física. Jones declara: "A coleção hipocrática é rica em palavras que significam delirium: 1) algumas nas quais o desarranjo mental é a ideia dominante e 2) algumas nas quais o acento está colocado sobre a fala delirante" (Hipócrates, 1972). Nesse ponto Jones está correto, já que o delirium tem sido considerado, desde essa época, resultante seja do prejuízo mental global ou de danos es- 
pecíficos ao "intelecto". No Corpus Hipocrático a associação mais frequente do delirium é com a frenite que (juntamente com a mania, a melancolia e a paranoia) constitui uma das quatro categorias da taxonomia psiquiátrica grega (Roccatagliata, 1973; Simon, 1978). Alguns historiadores da psiquiatria são anacrônicos quando supõem que estes três termos sejam coextensivos ao seu uso atual (Zilboorg, 1941; Alexander e Selesnick, 1966; Starobinski, 1962).

O conceito de frenite ilustra bem como os sintomas comportamentais e médicos se combinavam na medicina grega do século V a.C. (Garrison, 1929). Por exemplo, a ausência de febre era usada para separar a loucura convencional (como a mania) dos estados delirantes secundários à doença física. Esse critério permaneceu central na medicina ocidental até o início do século XIX (Cullen, 1785; Sutton, 1813; Middleton et al., cerca de 1780; Esquirol, 1814).

Sobre isso Von Feuchtersleben (1845) escreveu: “... a questão: se delirium e insanidade são idênticos... foi respondida assim: que o delirium agudo com febre deve ser distinguido da variedade crônica que é chamada de insanidade" (minha tradução). Observando a insuficiência desse critério de demarcação, o autor austríaco prosseguiu: “... mas a presença ou ausência de febre, que é possível em qualquer condição (não pode) decidir esse assunto". Ele propôs uma diferenciação sobre a base da história natural, sintomatologia e etiologia: "Delirium ... é um sintoma que indica a transição de uma doença puramente somática para um transtorno mental". Com relação à classificação variada de delirium, ele comentou: “... é infrutífero, como tem sido feito frequentemente, considerar diferenças no objeto (do delírio) como um terreno para a divisão... (pois)... eles não expressam a essência da perturbação" (minha tradução).

Concepções similares ao delirium podem também ser encontradas em outras culturas da antiguidade. Por exemplo, Hankoff (1972) examinou conceitos médicos no Talmud e identificou uma transliteração do termo grego Kordiakos, que era utilizada para referir uma loucura temporária associada ao consumo de vinho, provavelmente uma forma do delirium tremens.

\section{O século XIX}

Embora as observações psiquiátricas contidas na literatura clássica sejam epistemologicamente descontínuas com o presente, o historiador da psiquiatria deve analisá-las e se esforçar em destrinchar os acontecimentos psicológicos e comportamentais que elas contêm. Mais importante, ele precisa desvelar os sistemas conceituais que tornaram essas observações possíveis. Por exemplo, o fato de que no mundo pré-cartesiano nenhuma clara distinção ontológica fosse feita 
entre as doenças físicas e mentais significava que, na prática, nenhuma dificuldade descritiva ou etiológica surgisse quando as duas eram encontradas juntas. A aceitação no século XVII da separação cartesiana entre as substâncias extensa e pensante forçou a um giro conceitual nas teorias da insanidade, e a medicina ocidental começou a conjecturar sobre a associação entre a doença física e a doença mental (Kenny, 1968; Sauri, 1969; Lain Entralgo, 1978).

O século XIX, em resposta a isso, construiu sua própria epistemologia psiquiátrica (Swain, 1977), e testemunhou o desenvolvimento de uma psicologia que considerava a consciência como um constructo não material (Boring, 1950; Hamilton, 1859). Assim, os sintomas psicológicos se tornaram "significantes" de doença e foram concebidos como existindo em um domínio ontológico separado. A antiga observação de que mudanças mentais poderiam acompanhar doenças físicas criava, pela primeira vez, uma problemática teórica. Daí surgia a necessidade de: 1) uma descrição fenomenológica das próprias mudanças mentais; e 2) uma teoria da mediação que pudesse explicar os vínculos causais.

A história de como e por quais mecanismos a doença física pode produzir sintomas mentais deve distinguir três áreas de análise: em primeiro lugar, o desenvolvimento dos conceitos modernos de delirium e confusão; em segundo lugar, a formação da noção de psicose exógena; e em terceiro lugar, a metamorfose do termo demência. Neste artigo, apenas a primeira área será considerada.

O termo delirium se apresentava em Galeno como coextensivo a mentis alienatio (Siegel, 1973). Nessa forma ele permaneceu imutável até o século XIX. O dr. Johnson (1755) captou tanto seu sentido médico quanto não médico: ele definiu delírio como "insensatez, frenesi, senilidade". Sua citação de Swift ilustra bem os aspectos flutuantes e intelectualísticos do conceito: “... as pessoas disseram acerca dele que ele havia estado DELIRANTE por algumas horas, mas quando eu o vi, ele recuperara seu entendimento como eu sempre conheci". (Essa citação seria incorporada ao O.E.D. - Oxford English Dictionary um século depois). O dr. Johnson também definiu o delirium como "alienação da mente" e citou Arbuthnot: "Uma alacridade tão grande e prontidão de resposta, especialmente em pessoas naturalmente de outro temperamento, é um sinal de um DELIRIUM que se aproxima. Em um delirium febril há uma pequena inflamação do cérebro".

Desde os primeiros registros de seu uso (como de fato sua etimologia latina mostra) o significado de delirium tem sido associado com as perturbações no curso do pensamento. Essa interpretação intelectual (do que na prática é uma per- 
turbação comportamental generalizada) reinou soberana até o começo do século XIX (Middleton et al., cerca de 1780). Por exemplo, quando Sutton (1813) descreveu o conceito de delirium tremens, ou delirium com tremores, ele sentiu necessidade de contestar a antiga visão sobre a frenite: “À medida que a doença progride, as faculdades não se apresentam, geralmente, com qualquer extravagância de pensamento" (meu itálico). Ao incluir os distúrbios afetivos e motores em sua descrição, Sutton ampliou a concepção de delirium e minou a interpretação intelectualística.

Essa mudança teórica não ocorreu no vácuo. Desde o início do século XIX o associacionismo (a psicologia clássica do empirismo britânico) havia sido desafiado pela psicologia das faculdades mentais, uma perspectiva contida na psicologia de Wolf e na filosofia escocesa do senso comum, e popularizada pela frenologia (Spurzheim, 1826; Klein, 1970). A aceitação gradual da psicologia das faculdades mentais levou, na prática, a abordar a mente como um conjunto de funções autônomas. No seu devido curso, isso forneceu um novo quadro de classificações (Esquirol, 1838; Billod, 1848; Bucknill e Tuke, 1858).

$\mathrm{O}$ antigo duplo significado do delirium esteve presente durante o começo do século XIX, principalmente na psiquiatria francesa (Ball e Ritti, 1882). Pinel (1809), por exemplo, utilizou délire tanto para se referir a um erro específico de julgamento quanto para a frenite.

Esquirol (1814) adotou uma linha semelhante e desenvolveu uma visão do délire como uma perturbação perceptual, primariamente. "Uma pessoa é delirante quando suas ideias não estão em relação com suas sensações etc.”... "alucinações são a causa mais frequente de délire". No mesmo artigo, contudo, Esquirol alterna entre falar a respeito dos delírios e sobre o próprio delirium. Georget (1820) usou délire para se referir a transtornos do intelecto ou novas ideias. Ele de fato sugeriu como possíveis fontes para os délires: personalidade, pensamentos antagônicos e inteligibilidade (à la Jaspers) em termos de perturbação precipitante e origem bizarra ou mórbida. Paralelo a isso, ele também utilizou délire (como em délire aigu) para referir a "uma desordem nas funções intelectuais (e outras) resultantes de doença geral ou doença no cérebro" (Georget, 1820). Ele complementou essa definição com uma tabela detalhada mostrando o diagnóstico diferencial entre o délire aigu e a folie e assinalou a natureza sintomática, intermitente e reversível do primeiro. Em um artigo póstumo, Georget (1835) tomou uma perspectiva mais sindrômica, e separou o délire aigu (ou febril) do délire chronique ou sans fievre, que ele considerava equivalente à insanidade propriamente dita. Prejuízos a consciência, que viriam a constituir a distinção crucial durante a última parte do século XIX, ainda não estavam incluídos nestes escritos.

Por volta de 1860, o uso diferencial havia se estabilizado e délire estava sendo utilizado cada vez mais para se referir apenas às ideias aberrantes que acom- 
panham o delirium. Isso foi consolidado por Lasègue (1852), Falret (1864) e Magnan (Magnan e Sérieux, 1911) e legitimado no dicionário Littré (1877). O lapso deixado pelo uso específico do délire na psiquiatria francesa seria preenchido, após a metade do século, pelo termo confusão (confusion). Isso explica porque sua carreira na França tem sido mais duradoura do que, digamos, na psiquiatria inglesa, onde delírio e delirium foram adequadamente separados.

Igualmente na Alemanha não houve confusão terminológica e o delirium foi utilizado durante esse período para se referir à síndrome completa (Walther-Büel, 1973). A antiga palavra do alto alemão Wahn (loucura) sofreu um reajuste para acomodar a noção mais estreita e intelectualística de delírio (Ey, 1954).

Na Grã-Bretanha, o termo delírio foi usado desde o início daquele século para referir os transtornos da percepção (Conolly, 1830), após 1850, contudo, ele passou a descrever as "crenças errôneas" (Bucknill e Tuke, 1858; Clouston, 1887; Gowers, 1888).

Lipowski (1967), sem qualquer atenção à literatura francesa, declara: “A concepção de Tuke do delirium, que antecede o influente trabalho de Bonhoeffer, contém o núcleo da definição moderna dessa síndrome". Porém, o verbete que ele descreve foi provavelmente escrito por J.F.G. Pietersen, o superintendente do Asilo Ashwood House (que redigiu todas as pequenas definições, de A a M, no dicionário de Tuke (1892)), e foi "inspirado" por um verbete similar no Léxico de Medicina da New Sydenham Society (Power e Sedwick, 1882). De fato, o pequeno verbete de Pietersen (1892) não sumariza bem as perspectivas sobre o delirium por volta de 1890 . Por exemplo, não se refere às perturbações de consciência que, na época, eram amplamente aceitas tanto pelos autores ingleses quanto pelos do resto do continente.

Delirium versus Insanidade

Quando a medicalização das descrições psicopatológicas teve lugar durante o início do século XIX, delirium já era uma categoria estável (Middleton et al., cerca de 1780). Durante esse período, contudo, alguns autores colocaram em questão a distinção fundamental entre o delirium e as insanidades convencionais (Feuchtersleben, 1845; Brierre, 1845). Esse último era uma importante autoridade nesse assunto e em um longo e influente ensaio sobre o delirium agudo (1845) optou por uma visão sindrômica. Ele se baseou em algumas evidências estatísticas que mostravam, pelo menos para si próprio, que o delirium e as outras insanidades compartilhavam da mesma etiologia "moral"; e também em seus estudos post-mortem que ou falharam em encontrar quaisquer alterações patológicas ou 
encontraram apenas alterações inespecíficas. Ele perguntou, então, “será o caso que o delirium agudo seja apenas uma forma aguda da (insanidade convencional)?". Ele estudou 19 casos (11 descritos em seu artigo) dos quais apenas sete tiveram início agudo, nos restantes as perturbações haviam aparecido insidiosamente em um período de meses. Os casos três e nove eram de pacientes em estados clinicamente agudos de catatonia que, após recusarem persistentemente beber água ("hidrofobia sintomática", de acordo com Brierre), pareciam haver morrido de desequilíbrio eletrolítico e convulsões terminais. De modo semelhante ele incluiu os casos três, quatro, cinco, seis, sete e oito simplesmente devido à força de sua doença terminal, apesar de seu duradouro estado lipemaníaco (depressivo) pré-delirante. Essa amostra heterogênea o levou a colocar em questão a utilidade de critérios clínicos e patológicos e mesmo a presença de febre. Consequentemente, ele achou que não havia maneira de separar o delirium das outras insanidades. O critério do "estado de consciência", com seu concomitante sintoma de "desorientação temporoespacial" ainda não estava disponível. Isso só aconteceu após o termo "confusão" tornar-se popular durante a parte final do século XIX (veja abaixo).

As opiniões de Brierre tiveram influência. O American Journal of Insanity as patrocinou vinte anos depois e ofereceu uma sinopse favorável (Leader, 1864) sem, contudo, fornecer a referência! De modo semelhante, na Alemanha, Griesinger (1876) incluiu as opiniões de Brierre na segunda edição de seu manual. De fato, a questão de se o delirium agudo constituía uma forma separada de insanidade ou era apenas sua forma mais aguda prosseguiu até o final do século (Ball e Ritti, 1882; Gowers, 1888).

Entretanto, o afastamento de Brierre da perspectiva clássica não era amplamente aceito (Feuchtersleben, 1845). Dado que uma psicopatologia da consciência ainda não estava disponível, a única opção aberta para assegurar a organicidade do delirium era retornar ao estudo post-mortem. Calmeil (1859), em um trabalho volumoso, acusou os pesquisadores de desistir muito cedo e perder tempo com as diferenças sintomáticas. Ele reafirmou a natureza orgânica do delirium e concluiu que este era uma forma leve de encefalite e que, portanto, alterações patológicas de intensidade variável sempre poderiam ser encontradas. Outros assinalaram a baixa frequência do delirium tanto na prática privada quanto nos hospitais psiquiátricos (Thore, 1850).

O debate sobre o diagnóstico diferencial é muito relevante na história das psicoses. À medida que a visão anatomopatológica da doença ganhou popularidade na medicina, a antiga visão demonológica ou moral não era mais aceitável. A descoberta de Bayle $(1822,1825)$ de algumas mudanças patológicas reconhecíveis na paralisia geral dos alienados, satisfez apenas as necessidades etiológicas do novo modelo de doença, mas não ajudou a identificar padrões clínicos 
estáveis, que pudessem ser alinhados com as teorias das funções normais. Assim, uma reanálise do quadro clínico do delirium era muito mais útil para o desenvolvimento da psicopatologia do que a afirmação etiológica de Bayle. Na ocasião, o delirium era considerado, apesar de seu curso instável e flutuante, um microcosmo da insanidade, com mudanças patológicas afetando a cognição, as emoções e a ação. A declaração de Brierre, de que o delirium seria apenas uma forma aguda, prototípica, de insanidade, suportava essa hipótese. De fato, o próprio Calmeil, além de reafirmar uma diferença etiológica, não afirmou que o delirium e a insanidade pudessem ser sempre separados clinicamente. Assim, nas conclusões de seu capítulo sobre o delirium agudo, ele enumera sintomas compartilhados tanto pelo delirium quanto (pelo que ele denominava de) por insanidades funcionais.

Está fora do escopo desse artigo analisar a relevância desse assunto para a concepção da Einheits-psychose (psicose unitária), tal como foi desenvolvida por volta desse período na psiquiatria alemã (Llopis, 1954). Griesinger (1878) e Kahlbaum (1874), por exemplo, mantiveram uma visão unitária e alguns não psiquiatras, como Gowers (1888), também acreditaram que o delirium seria uma forma de insanidade com etiologia conhecida. Norman (1890), na Irlanda, sugeriu uma hipótese de continuum. Outros consideraram que isso não podia ser mais aceito (como Worcester, 1889).

Posteriormente no século, a separação do delirium das insanidades foi feita com base no envolvimento da consciência. Parece que esse critério se baseava menos na pesquisa empírica do que nos reajustes conceituais requeridos para explicar o estatuto e as características das insanidades remanescentes. Essas mudanças foram efetuadas em termos das teorias de doença então correntes (Sauri, 1972). A tradução de 1847 do livro de Feuchtersleben (1845) parece ter introduzido o termo "psicose" na psiquiatria inglesa. Contudo, o significado mais antigo de psicose, como um concomitante psicológico geral de qualquer mudança neurológica, permaneceu em uso até o final do século XIX (Baldwin, 1901). O fascinante processo pelo qual as psicoses se tornaram gradualmente orgânicas, enquanto as neuroses cruzavam, na direção oposta, para o polo psicológico, ainda não foi profundamente estudado pelos historiadores da psiquiatria. O livro de Lopez Piñero sobre as neuroses (1963) vai longe na explanação de suas transformações.

Durante as últimas duas décadas do século XIX, a transformação das insanidades nas psicoses foi consolidada por três inovações conceituais adicionais, duas das quais nós devemos a Magnan. Em primeiro lugar, sua noção de estabilidade genética explicou como certas formas de insanidade permaneciam verdadeiras seja intraindividualmente ou em gerações sucessivas; isso pôs um fim na visão de Morel da degeneração progressiva (1857; Baruk, 1967; Pistoia, 1973). Em segundo lugar, sua separação da insanidade com início agudo e bom prog- 
nóstico, da insanidade com um curso crônico conduzindo a eventual deterioração, ou seja, délire chronique (Magnan, 1886), pavimentou o caminho para uma perspectiva menos pessimista das psicoses. A terceira contribuição foi a opinião de Kraepelin de que a história natural de cada psicose deveria se tornar parte de sua definição.

\section{A consciência e suas perturbações}

Três mudanças caracterizam a transformação da psicologia em uma disciplina autônoma durante o século XIX: 1) a ênfase na análise dos conteúdos de consciência via psicofísica e introspecção; 2) a reconciliação do associacionismo clássico com a psicologia das faculdades mentais; 3) o estabelecimento de vínculos com a fisiologia e as técnicas de quantificação (Boring, 1950).

Relativo à natureza da consciência, o século XIX oscilou entre duas visões. Era considerada seja como uma função extra da mente (isto é, como uma entidade ontológica separada) ou como um epifenômeno, um tipo de consciência inerente a cada função mental (Hamilton, 1859). Necessidades clínicas e classificatórias tornaram a psiquiatria do século XIX crescentemente dependente das descrições dos conteúdos de consciência (Berrios, 1981). Esse giro, desde uma descrição comportamental para uma mentalística, criou a necessidade de unidades de análise dos atos e estados mentais. Essas foram providenciadas, durante a primeira metade do século, pela epistemologia combinatória do associacionismo. Daí, o comportamento insano tornou-se fragmentado em sintomas discretos (tais como alucinações, obsessões, delírios etc.), que no seu devido tempo viriam a ser considerados como o mobiliário básico da mente psicótica.

A incorporação da psicopatologia nas ciências médicas levou a uma reinterpretação do patológico como uma variação quantitativa do normal (Canguilhem, 1975; Foucault, 1954). Esse modelo de continuidade funcionava bem no caso de algumas unidades de análise, tal como as alucinações (Ey, 1973), mas não tão bem no caso de outras, como as obsessões, porque nenhum acordo podia ser alcançado quanto a qual faculdade mental estaria envolvida; as obsessões, portanto, resistiram à integração e foram consideradas como corpos estranhos (Berrios, 1977).

Essa estratégia de integração levou à análise complementar, qual seja, perguntar se todas as funções mentais poderiam desenvolver alterações patológicas. A consciência era uma dessas funções e desde que sua descrição incluía um "campo", um "foco central", uma "penumbra", suas alterações vieram a ser descritas como obscurecimento, constrição, turvação, opacidade, obliteração, clareza 
etc., isto é, como aquelas pertencentes a um tipo de suprapercepção autônoma. Essa visão era compatível com os princípios da psicologia das faculdades mentais e fornecia uma explicação para fenômenos clínicos como a despersonalização e a turvação (da consciência). Porém, por outro lado, a neurofisiologia desse período demandava que a cada função fosse dada, se possível, uma localização cortical: a consciência não era uma exceção para isso.

Os localizacionistas estavam divididos quanto a decidir se a consciência estaria situada no córtex cerebral ou em estruturas subcorticais (Davies, 1873). Esse debate teve lugar contra um pano de fundo de ideias religiosas e evolucionistas. Mas por volta de 1870 as contribuições de Carpenter, Maudsley, Jackson e Bastian inclinaram a balança a favor de uma localização centroencefálica (Walshe, 1957). $\mathrm{O}$ artigo de Bastian sobre a consciência (1870) montou a cena conceitual para a psiquiatria inglesa. O artigo de Wernicke Über das Bewusstsein (1879) fez o mesmo na psiquiatria alemã (Heimann, 1974).

A turvação de consciência tornou-se, durante a segunda metade do século XIX, o critério clínico para separar o delirium do resto das insanidades. A observação clínica de que os pacientes em delirium podem ser desorientados, obtusos, retardados e incapazes de lembrar suas experiências após a remissão foi documentada na literatura clássica (Dupuytren, 1834). Contudo, nenhuma tentativa havia sido feita no sentido de considerar esses sintomas como centrais no delirium, nem para explicá-los como resultantes de um prejuízo da consciência. A aceitação pela psicologia clássica de uma visão da consciência como uma função psicológica separada levou a psiquiatria a reinterpretar a desorientação e a confusão de ideias como perturbações dessa nova função.

\section{Parte II: Confusão}

O termo confusão persiste na psiquiatria inglesa (Fish, 1974) e foi entronizado na Classificação Internacional de Doenças como estado confusional agudo (OMS, 1978). Os franceses também mantiveram confusion mentale (Cottereau e Gaussel, 1971; Bernard e Trouvé, 1977) embora ela não tenha sido incluída na classificação INSERM de 1968 (Postel, 1972).

Verwirrtheit é infrequentemente utilizado na psiquiatria alemã e não mais diferenciado de Verworrenheit (Scarfetter, 1980). O DSM III (Associação Psiquiátrica Americana, 1980) e o PSE (Wing et al., 1974) derrubaram totalmente o termo. Autores descrevendo os estados orgânicos ou desconsideraram a confusão (Lipowski, 1980) ou a confinaram a um papel puramente descritivo (Lishman, 1978). 
O termo confusão tem sido empregado em linguagem jurídica desde os tempos de Roma, em lógica desde o período medieval e em epistemologia desde o século XVII (Eisler, 1904); em psicopatologia desde o século XIX (Grimm e Grimm, 1956). A confusão de ideias interpretada como aberração psicológica ganhou seu suporte teórico primitivo com o Associacionismo (Warren, 1921).

A confusão tem assim recebido tanto na lógica quanto na psicologia uma interpretação intelectual. Esquirol, por exemplo, usou-a para descrever um estado de perturbação intelectual ou caos, e J. S. Mill se referiu às falácias de confusão que resultavam da "concepção indistinta, indefinida e flutuante do que seja a evidência" (Mill, 1845). O barão Dupuytren (1834) a utilizou para descrever a desorientação que caracteriza o delirium nervoso.

Verwirrtheit, e os relacionados Verworren e Verwirren, também descreviam estados de caos mental e consequente perplexidade comportamental (Grimm e Grimm, 1956). Heinroth, Ideler e Spielman (Wille, 1888) usaram o termo para descrever estados de desordem mental e Griesinger (1878) estabeleceu seu estatuto nosológico. Wille, em seu clássico artigo, se referiu a ele como "perturbação funcional aguda, raramente crônica, do cérebro, caracterizada por confusão, alucinações, delírios, perturbação da consciência e algumas vezes estupor". Esse é o mesmo agrupamento de fenômenos mentais que Meynert (1890) descreveu como Amentia (Pappenheim, 1975). A influência do Associacionismo de Wundt é clara, tanto sobre Wille quanto Meynert. A confusão não figura como verbete nos dicionários de Tuke (1892) ou no de Power e Sedwick (1882).

O conceito psiquiátrico de confusão se desenvolveu durante o século XIX tanto na França quanto na Alemanha, para descrever um estado anormal, encontrado associado com o delirium ou as insanidades, e consistindo em um severo defeito na organização das ideias (Chaslin, 1892). De início ele se referia especificamente ao pensamento caótico e não implicava em etiologia orgânica. Daí, por pelo menos quarenta anos, foi aceito que a confusão poderia acompanhar qualquer forma de transtorno mental. De fato, apesar dos esforços de Chaslin, alguns autores (como Bleuler, 1911) ainda preferiam a antiga visão sindrômica.

\section{“Confusão" na psiquiatria francesa}

O termo "confusão" pode ser encontrado mencionado em relação ao delirium desde 1834 (Dupuytren, 1834), mas foi reintroduzido na psiquiatria francesa por Delasiauve (1851). Ele se opôs às opiniões de Baillarger sobre a identidade clínica entre a confusão e o estupor, declarando que a confusão lipemaníaca (isto é, depressiva) e a orgânica eram diferentes; e para explicar o aparente déficit 
cognitivo visto em algumas depressões, ele advogava um conceito similar ao da "demência pseudodepressiva". Delasiauve comparou a confusão com o devaneio, assim retornando ao "onirismo", uma hipótese presente na psiquiatria francesa pelo menos desde Baillarger (1843).

A confusão não entrou em uso corrente até avançado o século, mas o conceito, como Chaslin observou em 1892, tinha estado contido na noção germânica do Verwirrtheit. Esse atraso era devido ao sucesso da perspectiva de Baillarger de que a confusão seria uma forma de melancolia (Camuset, 1897). A contração gradual do conceito de melancolia conduziu ao aparecimento do estado confusional; Chaslin escreveu seu primeiro artigo sobre a matéria precisamente durante esse período. Apenas dois anos antes Conolly Norman publicara seu artigo sobre "A insanidade confusional aguda" (1890), contendo a maior parte dos elementos clínicos mais tarde expandidos pelo autor francês. O psiquiatra irlandês definiu a síndrome como tendo um rápido desencadear, prejuízo da consciência e experiências alucinatórias. Chaslin não deu muita atenção, ao menos abertamente, para o trabalho de Norman. A categoria sobreviveu até o século XX, por exemplo, em Bolton (1906) ou Bruce (1935), um dos palestrantes das Conferências Maudsley, que a estudou em seus acompanhantes físicos.

O primeiro artigo de Chaslin sobre a Confusão mental primitiva (1892) era uma versão expandida de uma comunicação escrita por ele, mas lida por Seglas no Congresso de Psiquiatria de Blois, no mês de agosto anterior. Desde o princípio, ele usou confusion (sob a influência de Wille) como equivalente a delirium, e em um sentido mais amplo do que o intelectualístico tradicional. Ele considerou a confusão mental um sintoma básico na esteira da qual as alucinações, o estupor, os delírios e os sintomas físicos poderiam seguir. Diferenciou-a de estados superficialmente similares que acompanham a mania, a melancolia, o délire chronique (isto é, esquizofrenia) e o delirium febril. Especulou que a confusão poderia ser devida à fraqueza cerebral.

$\mathrm{O}$ artigo de Chaslin fez soar a nota correta. Um ano mais tarde, no congresso psiquiátrico de La Rochelle, diversos estudos sobre a confusão foram apresentados, em apoio às suas ideias. Seglas (1894) em sua palestra na Salpêtrière reconheceu a contribuição de Chaslin e lamentou o fato de que o uso prévio de Delasiauve tivesse caído em desuso. Ele o atribuiu ao fato da persistente popularidade das ideias de Baillarger e de Morel. Também assinalou a relevância dos termos alemães Verwirrtheit, Amentia e Dysnoia, por eles se referirem "ao estrito senso do termo e não ao sintoma banal que acompanha todas as formas de insanidade, mas a um prejuízo muito específico... consistente na perda do controle voluntário sobre as funções intelectuais" (minha tradução).

A monografia de Chaslin apareceu em 1895. Nela, ele considerava a confusion mentale como resultante de automatismo psicológico, ou seja, da ca- 
tegoria explanatória que havia se tornado central na psicopatologia francesa após Ribot e Janet (Baruk, 1972). O automatismo psicológico se originaria da liberação de funções inferiores decorrentes da dissolução de funções superiores. $\mathrm{O}$ interesse de Ribot por Spencer o havia conduzido a Hughlings Jackson (Delay, 1953). O modelo hierárquico da mente que Chaslin emprestara de Ribot era, portanto, de origem jacksoniana. Sua perspectiva associacionista (resultante da influência de Wundt e Ribot) foi observada tanto por um revisor inglês (Brain, 1896) quanto por Camuset (1897), que confirmou o impacto do livro de Chaslin sobre a psiquiatria francesa. Outro revisor inglês (anônimo, 1898) achou que a confiança de Chaslin na teoria de Meynert era injustificada.

O termo adquiriu circulação internacional como resultado da contribuição de Régis e Hesnard (1911) para o "Tratado Internacional de Psicopatologia", de Marie. Eles identificaram três estágios na evolução do conceito: um primeiro período até 1843 , durante o qual a confusão, a demência e a stupidité estiveram fundidas (Berrios, 1981); um segundo período iniciando-se com o artigo de Baillarger (1843), durante o qual o estupor foi redefinido para incluir os estados confusionais; um terceiro período, começando com Delasiauve (1851) e complementado por Chaslin, Charpentier, Hannion, Seglas, Marandon de Montyel e outros, que consideraram a confusion mentale como uma síndrome separada.

Em seu próximo trabalho sobre o assunto, Chaslin (1915) abandonou de três maneiras sua posição original ao se referir à confusão como uma syndrome e não como uma maladie; ao descrevê-la como um transtorno global da função mental, e não apenas do intelecto; e ao sublinhar sua etiologia orgânica. Esse último ponto ele o fez no contexto de um debate com Eugen Bleuler, que ainda acreditava em uma visão sindrômica da confusão, e que havia criticado Anglade (um dos discípulos de Chaslin) por declarar que as verdadeiras síndromes confusionais (orgânicas) fossem diferentes da pseudoconfusão esquizofrênica (Bleuler, 1911). Régis (1906), em Bordeaux, também expressou um ponto de vista bleuleriano da mesma forma que Toulouse et al. (1920).

O debate francês sobre esse tema ocorreu em 1920 e foi compilado nas atas de três encontros sucessivos (de 29 de março a 31 de maio) da Société MédicoPsychologique (1923). Chaslin ali delineou, pela última vez, uma compreensão da confusão que perdura inalterada até hoje. Sua intervenção seguia a declaração de Toulouse et al. (1920) de que a confusão e a demência não seriam clinicamente diferenciáveis, porque ambas resultariam da mesma patologia, nomeadamente, um transtorno de "autocondução". Essa era uma categoria psicológica que gozou de alguma popularidade na França por volta da Grande Guerra e significava algo entre a autocondução e a auto-organização do ego. Referia-se àqueles aspectos voluntários e involuntários da personalidade que, em termos dinâmicos, preenchiam uma função adaptativa. Toulouse et al. também criticaram as técnicas de entre- 
vista sem estrutura utilizadas em psiquiatria na época, e as declararam muito cruas para detectar quaisquer diferenças diagnósticas que pudessem haver. Eles propuseram o uso de técnicas estandardizadas baseadas em questionários e análises de personalidade do tipo que Binet havia proposto alguns anos antes (Wolf, 1973; Binet, 1892).

Chaslin (1920) reiterou a opinião de que a perturbação psicológica crucial no estado confusional consistiria em um enfraquecimento da síntese, afetando as funções intelectual, afetiva e volitiva, e declarou que a autocondução de Toulouse era apenas outro nome para a antiga noção associacionista da síntese. Ele pensava que a origem do engano de Toulouse et al. não se derivava de qualquer similaridade clínica ou patogênica entre a confusão e a demência, mas da moda recentemente desenvolvida de usar demência como uma ferramenta prognóstica. Ele alertava que o prognóstico era incerto quando feito com base em exames transversais. De fato, um dos temas centrais no debate de 1920 era se uma análise transversal do estado mental era suficiente para o diagnóstico e o prognóstico. Toulouse et al. postularam que era assim, se corretamente conduzido. Chaslin defendeu uma abordagem histórica.

Uma consequência do debate de 1920 foi que a confusão mental também ficou vinculada com a turvação ou obliteração da consciência (Porot, 1975). Isso a fez efetivamente sinônima do delirium; donde os esforços posteriores para separá-los, seja em termos da severidade ou tipo de funções mentais envolvidas, estavam destinados ao fracasso na medida em que ambos os conceitos se referiam ao mesmo fenômeno biológico. A única diferença era teórica: a confusão se referindo à incoerência de ideias; o delirium aos transtornos perceptivos e motores.

\section{Consequências}

Com Kurt Schneider (1948; Conrad, 1960), a turvação se tornou "axial” para a escola alemã; sua contrapartida clínica sendo a desorientação temporoespacial. O uso simplista dessa noção tem frequentemente ocultado sua complexidade (Levin, 1956; Benton et al., 1964; Lipowski, 1980; Marchais, 1981). A urgência de alcançar um entendimento da desorientação é determinada pelo fato de que ela carrega, ao menos na psiquiatria anglo-saxã, o peso de ser o concomitante clínico da "turvação", uma descrição metafórica ainda considerada como a característica fundamental do delirium (Lipowski, 1980).

A observação arguta de Chaslin de que estados orgânicos agudos podem ocorrer sem turvação, foi recuperada pela escola alemã quando, na década de 
1950, ela reconheceu a síndrome transicional ou Durchgang (Wieck, 1961) como uma psicose sintomática reversível sem turvamento de consciência. Isso foi confirmado por autores europeus (Alonso Fernandez, 1977). De modo interessante, essa opinião influenciou as categorias nosológicas relevantes no DSM III (ou seja, síndromes delirante ou afetiva orgânicas).

Está além dos limites temporais desse artigo explorar o caminho no qual a evolução do delirium, confusão e turvação de consciência convergiram no trabalho de Bonhoeffer (1910) e relacionou-se com a polêmica questão da falsa dicotomia exógeno-endógeno. Do que já foi dito, pode ser conjecturado que eu acredito que a contribuição de Bonhoeffer foi de algum modo supervalorizada, e que os assuntos conceituais cruciais envolvidos nos estados orgânicos já haviam sido resolvidos por volta do final do século XIX. A superestimação dos resultados de Bonhoeffer deriva de uma interpretação histórica muito linear e de uma completa desconsideração pelas fontes francesas (Bleuler et al., 1966; Zutt et al., 1969). Talvez uma explicação da rápida aceitação, pós 1910 , da visão estereotipada de Bonhoeffer, do delirium e síndromes assemelhadas, possa ser encontrada na qualidade de sua monografia, ou mesmo no prestígio de Aschaffenburg. Isso não é muito plausível porque a monografia de Redlich (1912), na mesma série, é muito mais impressionante do que a de Bonhoeffer.

Uma melhor explanação é sugerida pela evolução dos próprios conceitos. A aquisição de seus próprios princípios organizacionais pelas psicoses funcionais no mundo pós-kraepeliano levou a uma negligência conceitual do delirium e da confusão, na medida em que elas não mais forneciam um paradigma para as psicoses e nem, de fato, se definiam em detalhes psicopatológicos. Consequentemente, a visão estereotipada se tornou suficiente (evidências para isso serão trazidas em um artigo futuro).

\section{Sumário e Conclusões}

Os estados orgânicos agudos em psiquiatria com as categorias relacionadas como o delirium e a confusão são importantes dos pontos de vista teórico e clínico. Estudos históricos adequados sobre a avaliação desses fenômenos são necessários antes que descrições históricas mais complexas sejam tentadas.

Os seguintes pontos foram assinalados:

1) Mudanças no conceito de delirium durante a primeira metade do século XIX resultaram da separação da noção mais estreita de délire (delírio).

2) Delirium foi redefinido pela nova psicopatologia como uma perturbação da consciência, seus sintomas acompanhantes sendo considerados como secun- 
dários ou fenômenos derivados, e sua etiologia como orgânica em um sentido forte.

3) Essa redefinição do delirium conduziu à sua separação bem-sucedida das insanidades funcionais, assim acelerando a transformação conceitual dessas últimas nas psicoses.

4) "Confusão" é uma contribuição do século XIX. Em sua origem ela constituía uma noção intelectual estreita, atribuída como resultante de uma perturbação da síntese. Consequentemente, ela poderia acompanhar tanto os estados orgânicos como os funcionais.

5) Confusion sobreviveu na psiquiatria francesa porque ela preencheu um papel conceitual, o mesmo que o delirium ocupou na psiquiatria inglesa.

6) Do ponto de vista histórico, o conceito de confusão ilustra melhor do que o de delirium os esforços dos psiquiatras do século XIX em elucidar a disfunção psicológica que caracteriza os estados orgânicos agudos.

7) O conceito de turvação desenvolvido como um produto das perspectivas do século XIX sobre a consciência, provê o delirium de um sintoma nuclear muito mais flexível do que a interpretação intelectual da confusão. A desorientação temporoespacial provou-se uma característica clínica não confiável para assegurar a presença da turvação.

8) O conceito de confusão e o mais recente, de síndrome transicional, podem adquirir nova utilidade se reinterpretados em termos da psicologia da cognição do século XX e adequadamente confirmados por evidência empírica.

\section{Referências}

ACKnerknecht, E. H. Kurze Geschichte der Psychiatrie. Stuttgart: Enke Verlag, 1957. English translation by S. Wolff. A Short History of Psychiatry. New York and London: Hafner, 1959.

Alexander, F.; Selesnick, S. The History of Psychiatry. New York: Harper \& Row, 1966. Alonson Fernández, F. Fundamentos de la psiquiatria actual. Madrid: Editorial Paz Montalvo, 1977. v. 2, p. 603-4.

American Psychiatric Association. Diagnostic and Statistical Manual of Mental Disorders. 3. ed. Washington, 1980.

Anonymous. Reviews and Abstracts. Brain, n. 19, p. 123-7, 1896.

. Review of Chaslin. Journal of Mental Science, n. 44, p. 159-62, 1898.

Baillarger, M. J. De l'etat désigné chez lcs aliénés sous le nom de stupidité. Annales Medico-psychologiques, n. 1, p. 76, 256, 1843. 
Baldwin, J. M. Dictionarv of Philosophy and Psychology. London: Macmillan, 1901. v. 2.

Ball, B.; Ritti, A. Délire. In: Dechambre, T. (Ed.). Dictionaire Encyclopédique des Sciences Médicales. Paris: P. Asselin, S. R. de Labé and Victor Masson et fils, 1882. v. 26.

Baruk, H. La Psychiatrie Française de Pinel à nos Jours. Paris: Presses Universitaires de France, 1967.

. Automatisme et troubles des mécanismes de la pensée intérieure dans la psychiatric francaise et dans la psychologie interprétative de Freud. In: B ASTIDE, R. (ed.). Les Sciences de la Folie. Paris: Mouton, 1972.

Bastian, H.C. Consciousness. Journal of Mental Science, n. 15, p. 501-23, 1870.

BAYLe, A.L.J. Recherches sur l'Arachnitis Chronique ... Considérées comme Cause d'Aliénation Mentale. Paris: Gabon, 1822.

. Nouvelle Doctrine des Maladies Mentales. Paris: Gabon, 1825.

Benton, A.L.; Van Allen, M.W.; Fogel, M.L. Temporal disorientation in cerebral disease. Journal of Nervous Mental Disease, n. 139, p. 110-19, 1964.

Bernard, P.; Trouvé, S. Sémiologie Psychiatrique. Paris: Masson, 1977.

Berrios, G. E. Henri Ey, Jackson et les idées obsédantes. L' Evolution Psychiatrique, n. 42, p. 687-99, 1977.

. Stupor, a conceptual history. Psychological Medicine, 1981 (in press).

Billod, E. Maladies de la volonté. Annales Médico-psychologiques, n. 10, p. 15-35, 170-202, 317-47, 1847.

Binet, A. Les Altérations de la Persoualité. Paris: Alcan, 1892.

Bleuler, E. Dementia Praecox oder die Gruppe der Schizophrenien. In:

Aschaffenburg, G. (Ed.). Handbuch der Psychiatrie. Leipzig: Deuticke, 1911. English translation by J. Zinkin. Dementia Praecox or the Group of Schizophrenias. New York: International Universities Press, 1951.

Bleuler, M.; Willi, J.; Buhler, H.R. Akute Psychische Begleiterscheinungen Körperlicher Krankheiten. Stuttgart: George Thiem Verlag, 1966.

Bolton, J.S. Amentia and dementia: a clinico-pathological study. Journal of Mental Science, n. 52, p. 427-90, 1906.

Bonhoefrer, K. Die Symptomatischen Psychosen. Leipzig: Franz Deuticke, 1910.

Boring, E.G. A History of Experimental Psychology. 2. ed. New York: AppletonCentury Crofts, 1950.

Brierre de Boismont, A. Du délire aigu. Memoires de l'Academie de Médecine, n. 11, p. $477-595,1845$.

Rev. Latinoam. Psicopat. Fund., São Paulo, v. 14, n. 1, p. 166-189, março 2011 
BruCE, L.C. Physical symptoms of acute confusional insanity.Lancet, n. i, p. 550-1, 1935. Bucknill, J.C.; Tuke, D. H. A Manual of Psychological Medicine. London: John Churchill, 1858.

Calmeil, L.F. Traité des Maladies Inflammatoires du Cerveau. Paris: J. B. Baillière et Fils, 1859. $2 \mathrm{v}$.

CAmuset. Review of Chaslin's "La confusion mentale primitive". Annales Médicopsychologiques, 8th Series, n. 5, p. 317, 1897.

Canguilhem, G. Le Normal et le Pathologique. Paris: Presses Universitaires de France, 1975.

Chaslin, PH. La confusion mentale primitive. Annales Médico-psychologiques, 7th Series, n. 16, p. 225-73, 1892. . La Confusion Mentale Primitive. Paris: Asselin et Houzeau, 1895.

43,1915 . . La confusion mentale. Annales Medico-psychologiques, n. 6, p. 276-89, 413-

. Quelques mots sur la confusion mentale. Annales Medico-psychologiques, $\mathrm{n}$. 6, p. 356-66, 1920.

Clouston, T.S. Clinical Lectures on Mental Disease. London: J. \& A. Churchill, 1887.

Conolly J. All Enquiry Concerning the Indications of Insanity. London: John Taylor, 1830.

Conrad, K. Die symptomatischen Psychosen. In: Grühle, H.W.; Mayers-Gross, W. (eds.). Psychiatrie der Gegenwart. Berlin: Springer Verlag, 1960.

Cotterau, M.; Gaussel, J. Syndrome confusionnel. Revue de Médecine, n. 27, p. 1739-43, 1971.

Cullen, W. Synopsis Nosologiae Methodicae. Edinburgh: Gulielums Creech, 1785. v. 3, p. 90 and 254.

DAviES, W.G. Consciousness and 'unconscious cerebration'. Journal of Mental Science, n. 18, p. 202-17, 1873.

Delasiauve, M. Du diagnostic différentiel de la lypémanie. Annales Médicopsychologiques, 2nd Series, n. 3, p. 380-442, 1851.

Delay, J. Le Jacksonisme et l'oeuvre de Ribot. In: Études de Psychologie Medicale. Paris: Presses Universitaires de France, 1953.

Dupuytren, Baron De. On nervous delirium. Lancet, n. i, p. 919-23, 1834.

EISLER, R. Wörterbuch der Philosophischen Begriffe. Berlin: Mittler und Sohn, 1904. v. 2. EsQuirol, J.E.D. Délire. In: Dictionnaire des Sciences Médicales. Paris: C.L.F. Panckoucke, 1814. 
. Des Maladies Mentales. Paris: J. B. Baillière, 1838.

Ey, H. Confusion et délire confuso-onirique. Étude 24. In: Etudes Psychiatriques. Paris: Desclée de Brouwer, 1954. v. III. . Traité des Hallucinations. Paris: Masson, 1973.

Falret, J.P. Délire. In: Des Maladies Mentales et des Asiles d'Aliénés. Paris: P. Asselin SR de Labe, 1864. p. 354.

Feuchtersleben, E. von. Lehrbuch der Ärztlichen Scelenkunde. Vienna: Carl Gerold, 1845. p. 228-29. English translation by H. E. Lloyd. The Principles of Medical Psychology. London: New Sydenham Society, 1847.

Fish, F. Clinical Psychopathology. (Revised by Max Hamilton). Bristol: John Wright, 1974.

Foucault, M. Maladie Mentale et Psychologie. Paris: Presses Universitaires de France, 1954. English translation by Alan Sheridan. Mental Illness and Psychology. New York: Harper Colophon Books, 1976.

Garrison, F.H. An Introduction to the History of Medicine. Philadelphia: W. B. Saunders, 1929.

Georget, E.J. De la Folie. Paris: Crevot, 1820. . Délire. In: Dictionnaire de Médecine ou Répertoire Général des Sciences Médicales. 2. ed. Paris: Bechet, 1835. v. 10.

Gowers, W. A Manual of Diseases of the Nervous System. London: J. \& A. Churchill, 1888. v. 2, p. 97.

Griesinger, W. Die Pathologie und Therapie der Psychischen Krankheiten.

Braunschweig: Friedrich Wroden, 1878. English translation of second German edition by C. Lockhart Robertson and J. Rutherford. Mental Pathology and Therapeutics. London: New Sydenham Society, 1867.

Grimm, J.; Grimm, W. Deutsches Worterbuch. Leipzig: S. Hirzel, 1956. v. 12.

Hamilton, W. Lectures on Metaphysics and Logic. Edinburgh: William Blackwood \& Sons, 1859. v. I, p. 129-383.

HANKoff, L.D. Ancient descriptions of organic brain syndrome: the 'Kordiakos' of the Talmud. American Journal of Psychiatry, n. 129, p. 233-6, 1972.

Heimann, H. Bewusstseinsstörungen. In: MüLler, C. (Ed.). Lexicon der Psychiatrie. Berlin: Springer Verlag, 1974.

HipPocRATES. Works. (with an English translation by W.H.S. Jones). Loeb Classical Library. London: William Heinemann, 1972. v. I.

Johnson, S. A Dictionary of the English Language. London: J. and P. Knopton, T. Longman et al, 1755. 
Kahlbaum, K.L. Die Katatonie. Berlin: Kirschwal, 1874. English translation by Y. Levi and T. Pridan. Catatonia. Baltimore: John Hopkins University Press, 1973.

Kenny, A. Descartes: A Study of his Philosophy. New York: Random House, 1968.

KLEIN, D.B. A History of Scientific Psychology. London: Routledge \& Kegan Paul, 1970. p. 638-98.

Lain Entralgo, P. Historia de la Medicina. Barcelona: Salvat, 1978.

Lasègue, C. Du delire de persécution. Archives Generales de Médicine, n. 28, p. 129$50,1852$.

LEADER. Acute delirium in 1845 and 1860. American Journal of Insanity, n. 21, p. 181200, 1864.

Leibrand, W.; Wettley, A. Der Wahnsinn, Geschichte der Abendländischen Psychopathologie. Freiburg: Karl Alber, 1961.

LeVIN, M. Varieties of disorientation. Journal of Mental Science, n. 102, p. 619-23, 1956.

Lipowski, Z.J. Delirium. Acute Brain Failure in Man. Springfield, Illinois: Charles C. Thomas, 1980.

Lishman, W.A. Organic Psychiatry. Oxford: Blackwell, 1978.

LitTRÉ, E. Dictionnaire de la Langue Française. Paris: Librairie Hachette, 1877. v. 2.

Llopis, B. La psicosis unica. Archivos de Neurobiologia, n. 17, p. 1-39, 1954.

LÓPEZ Piñero, J.M. Origenes historicos del concepto de neurosis. Cuadernos Valencianos de Historia de la Medicina, 1963.

Magnan, V. Considérations générales sur la folie. Le Progrès Médical, n. 4, p. 108991,1108-12; n. 5, p. 187-90, 209-13, 1886-7.

Magnan, V.; Serieux, T. Délire chronique. In: Traité International de Psychologie Pathologique. (Directeur A. Marie). Paris: Librairie Félix Alcan, 1911. v. 2.

Marchais, P. Les Processus Psychopathologiques de l'Adulte. Paris: Privat, 1981

Meynert, T.H. Amentia, die Verwirrtheit. Jahrbücher für Psychiatrie und Neurologie, n. 9, p. 1, 1890.

Middleton, E.; Turnbull, W.; Ellis, T.; Davison, J. Dictionary of Arts and Sciences; or A Universal System of Useful Knowledge. London: Alex Hog, (circa 1780).

Mill, J.S. A System of Logic (1898 Ed.). London: Longmans, Green \& Co., 1845.

Morel, B.A. Traité des Dégénérescences Physiques Intellectuelles et Morales de l'Espèce Humaine. Paris: J. B. Baillière, 1857.

Norman, C. Acute confusional insanity. Journal of Medical Science, Dublin, n. 89, p. 506-18, 1890 . 
Pappenheim, E. On Meynert's amentia. International Journal of Neurology, n. 9, p. 310-26, 1975.

Pietersen, J.F.G. Delirium. In: Tuke, D.H. (Ed.). Dictionary of Psychological Medicine. London: Churchill, 1892. p. 332.

Pinel, P. Traité Medico-philosophique sur l'Alienation Mentale. 2. ed. Paris: J. Ant. Brosson, 1809.

Pistoia, L. Del. Le problème de la ternporalité dans la psychiatrie française classique. L'Evolution Psychiatrique, n. 36, p. 445-74, 1973.

Porot, A. Manual Alphabetique de Psychiatrie. Paris: Presses Universitaires de France, 1975.

Postel, J. (ed.). Introduction (p 7-21) to selected texts, De la Folie. (Georget, E. 1820). Paris: Privat, 1972.

Power, B.; Sedwich, L.W. The New Sydenham Society's Lexicon of Medicine and the Allied Sciences. London: New Sydenham Society, 1892.

Redlich, E. Die Psychosen bei Gehirnerkrankungen. In: Aschaffenburg, G. (Ed.). Handbuch der Psychiatrie. Leipzig: Franz Deuticke, 1912.

RéGIs, E. Précis de Psychiatrie. Paris: Octave Doin, 1906.

RéGis, E.; Hesnard, A. Les confusions mentales. In: Marie, A. (Ed.). Traité

International de Psychopathologie. Paris: Felix Alcan, 1911. v. 2.

Roccatagliata, G. Storia della Psichiatria Antica. Milan: Ulrico Hoepli Editore, 1973.

SAURI, J.J. Historia de las Ideas Psiquiátricas. Buenos Aires: Carlos Lohle, 1969. . Las significaciones del vocablo psicosis. Acta Psiquiatrica Psicologica de America Latina, n. 18, p. 219, 1972.

SCharfetter, C. General Psychopathology. Cambridge University Press, 1980.

SCHNeider, K. Beiträge zur Psychiatrie. Stuttgart: Enke Verlag, 1948.

Seglas, J. De la confusion mentale primitive. Archives Générales de Médécine, n. 1, p. 538-49, 665-84, 1894.

Siegel, R.E. Galen on Psychology, Psychopathology and Function and Diseases of the Nervous System. Basel: S. Karger, 1973.

Simon, B. Mind and Madness in Ancient Greece. London: Cornell University Press, 1978.

Société Médico-Psychologique (1923) Annales Medico-psychologiques, 1923. p. 288.

Spurzheim, G. Phrenology in Connexion with the Study of Physiognomy. London:

Treuttel, Wurtz and Richter, 1826.

StARobinski, J. Historia del tratamiento de la melancolia de los orígenes hasta 1900. Acta Psychosomatica n.3. Basel: Geigy S.A., 1962. 
Sutton, T. Tracts on Delirium Tremens, on Peritonitis . . . and on the Gout. London: Thomas Underwood, 1813. p. 1-77.

Swain, G. Le Sujet de la Folie, Naissance de lu Psychiatrie. Paris: Privat, 1977.

THoré, M. fils. De la folie consécutive aux maladies aiguës. Annales Medicopsychologiques, 2nd Series, n. 2, p. 586-602, 1850.

Toulouse; Juquelier; Mignard. Confusion, démence et autoconduction. Annales Medico-Psychologiques, 10th Series, n. 12, p. 335-49, 1920.

TuKe, D.H. (Ed.). Dictionary of Psychological Medicine. London: Churchill, 1892. 2 v., p. viii.

Walshe, F.M.R. The brain stem conceived as the 'highest level' of function in the nervous system, with particular reference to the 'automatic apparatus' of Carpenter (1850) and to the 'centrencephalic integrating system' of Penfield. Brain, n. 80, 51039, 1957.

W ALther-Büel, H. Delirien. In: Lexicon der Psychiatrie (Muller, C. Herausg.). Heidelberg: Springer Verlag, 1973.

W ARREN, H.C. A History of the Association Psychology. New York: Charles Scribner, 1921.

Wernicke, C. Uber das Bewusstsein. Allgmeine Zeitschrift für Psychiatrie, n. 35, p. $420,1879$.

WIECK, H.H. Zur klinische Stellung des Durengangs- yndrome. Schweizer Archiv für Neurologie und Psychiatrie, n. 88, p. 409-19, 1961.

WILLE, L. Die Lehre von der Verwirrtheit. Archives für Psychiatrie, n. 19, p. 328-51, 1888.

Wing, J.K.; COOPER, J.E.; SARTORIUs, N. The Measurement and Classification of Psychiatric Symptoms. Cambridge University Press, 1974.

Wolf, T.H. Alfred Binet. Chicago and London: The University of Chicago Press, 1973.

Worcester, W.L. Delirium. American Journal of Psychiatry, n. 46, p. 22-7, 1889.

World Health Organization. Mental Disorders: Glossary and Guide to their Classification in accordance with the Ninth Revision of the International Classification of Diseases. Geneva: WHO, 1978.

Young, R.M. Mind, Brain and Adaptation in the 19th Century. Oxford: Clarendon Press, 1970.

Zilboorg, G. A History of Medical Psychology. New York: W. W. Norton \& Co., 1941.

Zutt, J.; Straus, E.; Schiller, H. (Eds.). Karl Bonhoeffer, Zum Hundertsten

Geburstag am 31 Mars 1969. Berlin: Springer-Verlag, 1969. 


\section{Resumos}

(Delirium and confusion in the 19th century: a conceptual history)

Delirium remained a stable psychiatric category until the early 19th century when it underwent aetiological and phenomenological redefinition, precipitating the transformation of the functional insanities into psychoses. Confusion, introduced by the French during the second half of the century, referred to a syndrome wider than (but including) delirium. It emphasized chaotic thinking and cognitive failure. The notion of clouding of consciousness (and tempora-spatial disorientation) established a common denominator for the two concepts, while Chaslin and Bonhoeffer redefined confusion and delirium asthe stereotyped manifestations of acute brain failure.

Key words: Delirium - history, mental confusion - history, disorders of conscience history, cognitives disorders - history

(Le délire et la confusion au XIX ${ }^{\mathrm{e}}$ siècle: une histoire conceptuelle)

Le délire était une catégorie psychiatrique stable jusqu'au début du XIXe siècle. Elle subi alors une redéfinition étiologique et phénoménologique précipitant la transformation des insanités fonctionnels dans les psychoses. La confusion, introduite par les français au cours de la seconde moitié du siècle, renvoie à un syndrome plus ample que le délire (et l'inclut). Elle met en relief la pensée chaotique et les échecs cognitifs. La notion de trouble de la conscience (et de la désorientation temporospatiale) a établi un dénominateur commun pour les deux concepts, tandis que Chaslin et Bonhoeffer ont redéfini la confusion et le délire comme des manifestations stéréotypées de la défaillance cérébrale aiguë.

Mots clés: Delirium - histoire, confusion mentale - histoire, troubles de la conscience histoire, troubles cognitifs - histoire

(El delirium y la confusión en el sigo XIX: una historia conceptual)

El delirium permaneció una categoría psiquiátrica estable hasta inicios de siglo XIX, cuando pasó por una redefinición etiológica e fenomenológica, precipitando la transformación de las insanias funcionales en psicosis.

La confusión, introducida por los franceses a lo largo de la segunda mitad de siglo, se refería a un síndrome más amplio (pero incluyendo) o delirium. Enfatizaba el pensamiento caótico y las fallas cognitivas. La noción de turbación de la conciencia (y desorientación temporal-espacial) estableció un denominador común para las dos concepciones, en cuanto Chaslin y Bonhoeffer redefinieron la confusión y el delirium como as manifestaciones estereotipadas de la insuficiencia cerebral aguda.

Palabras-claves: Delirium - historia; confusión mental - historia; trastornos de la conciencia - historia; trastornos cognitivos - historia 
Citação/Citation: Berrios, G.E. Delirium e confusão mental no século XIX: uma história conceitual. Revista Latinoamericana de Psicopatologia Fundamental, São Paulo, v. 14, n. 1, p. 166189, mar.2011.

Editor do artigo/Editor: Prof. Dr. Manoel Tosta Berlinck

Recebido/Received: 6.7.2010/ 7.6.2010 Aceito/Accepted: 25.9.2010 / 9.25.2010

Copyright: (C) 2009 Associação Universitária de Pesquisa em Psicopatologia Fundamental/ University Association for Research in Fundamental Psychopathology. Este é um artigo de livre acesso, que permite uso irrestrito, distribuição e reprodução em qualquer meio, desde que o autor e a fonte sejam citados/This is an open-access article, which permits unrestricted use, distribution, and reproduction in any medium, provided the original author and source are credited.

Financiamento/Funding: O autor declara não ter sido financiado ou apoiado/The author has no support or funding to report.

Conflito de interesses/Conflict of interest: $\mathrm{O}$ autor declara que não há conflito de interesses/The author declares that has no conflict of interest.

\section{GERMAN E. BerRios}

Médico e filósofo pela Universidad Nacional de San Marcos, Lima, Peru; Psiquiatra; Neurologista; Psicólogo; Filósofo; Historiador e Filósofo da ciência (Oxford University, England); Professor de Neuropsiquiatria e de Epistemologia da Psiquiatria (University of Cambridge, England), desde 1976; Neuropsiquiatra e Chefe do Departamento de Neuropsiquiatria do Hospital Addenbrooke, University of Cambridge, por 32 anos; Coordenador do Comitê de Ética em Pesquisa com Humanos na mesma universidade, por 20 anos; Editor Responsável de History of Psychiatry; Autor de 14 livros, incluindo The History of Mental Symptoms, Descriptive Psychopathology since 19th Century (Prêmio Nacional BMA, 1997), A History of Clinical Psychiatry (com Roy Porter), e Delirio (com F. Fuentenebro) e mais de 400 artigos e capítulos de livros; Membro do Royal College of Psychiatrists; da Associação Britânica de Psicologia e da Academia Britânica de Ciências Médicas; Membro Vitalício do Robinson College, Cambridge; doutor Honoris-Causa da Universidade de Heidelberg (Alemanha), da Universidade Nacional Mayor de San Marcos (Peru) e da Universidad Autónoma de Barcelona (Espanha); Grão Oficial da Ordem del Sol (Condecoração do Governo Peruano, 2007); prêmio Ramon y Cajal 2008 concedido pela Asociación Internacional de Neuropsiquiatria.

University of Cambridge

Box 189, Hills Road

Cambridge,

UK CB2 2QQ

e-mail: geb11@cam.ac.uk 